\title{
MULTIPLE-BAND ENVELOPE-FUNCTION APPROXIMATION FOR THE MAGNETOOPTICAL ANISOTROPY OF EXCITONS IN STRAINED SEMIMAGNETIC SEMICONDUCTORS
}

\author{
D. Suisky, F. Neugebauer, J. Röseler and S. Rex \\ Institute of Theoretical Physics, Humboldt-University Berlin \\ Invalidenstr. 110, D-O-1140 Berlin, Federal Republic of Germany
}

The magnetooptical anisotropy in strained quantum well structures is studied theoretically. As the source of this anisotropy the magnetic field induced mixing of light and heavy hole states is found. The anisotropy is also found in the frame of the multiple-band envelope-function approximation studying the influence of the barrier material on electronic states in the quantum well.

PACS numbers: 75.70.Ak, 73.20.Dx

\section{Introduction}

Semimagnetic semiconductors have attracted a great deal of interest as model systems for fundamental research and applications. Quantum wells (QW) and superlattices (SL) made up of CdTe and CdMnT exhibit due to exchange interaction between the spins of the paramagnetic Mn electrons and the valence or conduction band electrons new properties. They are characterized by a combination of the semiconductor band structure properties with the special features of the half-filled $3 d$-shell of the Mn ions.

The mixed crystal behavior of usual semiconductor bulk materials is modified if different materials are combined in growing single $Q W$, double heterostructures (DHS) or SL. The lattice misfit causes a biaxial build-in strain which modifies the electronic states in the QW as well as in the barrier. The main result is a reduction of the cubic symmetry to a tetragonal symmetry.

In our paper we will give a theoretical description of the different influence of the magnetic field parallel and perpendicular to the QW layer. We confirm the interpretation of Goede et al. [1,2] which was given recently for explanation of the observed strong anisotropy of the barrier cap excitons on the basis of the Gubarev model [3]. We extend the calculetions to the transitions in the QW using the envelope-function approximation (EFA) for studying the interplay of strain and magnetic field for different intensities of the magnetic field. 


\section{The interplay of the magnetic field and the build-in strain}

The Gubarev model was formulated for hexagonal materials [3] and applied to $\mathrm{CdSe} / \mathrm{CdMnSe}[1,2]$ in the cubic approximation [4] by reinterpretation of the crystal field splitting constant as the strain induced contribution.

Using the basis set

$x \alpha, \mathrm{i} y \beta, z \beta, x \beta,-\mathrm{i} y \beta,-z \alpha$

the Hamiltonian reads [4]

$$
H=H_{\mathrm{kp}}+H_{\mathrm{so}}+H_{\mathrm{ex}}+H_{\mathrm{str}}+H_{\mathrm{mag}} \text {. }
$$

The first term describes the band structure in kp-approximation, the second - the spin-orbit interaction, the third - the exchange interaction in the Kossut-Gałazka form [5]. Furthermore, we include the contribution due to the build-in strain. The last term in (2) describes the direct influence of the magnetic field on the band states being small in comparison with $H_{\mathrm{ex}}$.

Diagonalizing $H_{\text {so }}$ one obtains the $\Gamma_{8}$ valence states and the spin-orbit split band. The strain-induced contribution for the biaxial deformation follows from:

$$
\begin{aligned}
H_{\mathrm{str}} & =\left[\begin{array}{cc}
T_{\mathrm{C}}^{\mathrm{str}} & 0 \\
0 & T_{\mathrm{C}}^{\mathrm{str}}
\end{array}\right], \\
T_{\mathrm{C}}^{\mathrm{str}} & =\left[\begin{array}{ccc}
l \varepsilon_{x x}+\left(\varepsilon_{y y}+\varepsilon_{z z}\right) m & n \varepsilon_{x y} & n \varepsilon_{x z} \\
n \varepsilon_{x y} & l \varepsilon_{y y}+\left(\varepsilon_{x x}+\varepsilon_{z z}\right) m & n \varepsilon_{y z} \\
n \varepsilon_{x z} & n \varepsilon_{y z} & l \varepsilon_{z z}+\left(\varepsilon_{x x}+\varepsilon_{y y}\right) m
\end{array}\right]
\end{aligned}
$$

choosing $\varepsilon_{x y}=\varepsilon_{x z}=\varepsilon_{y z}=0$. Here $l, m$ and $n$ are the deformation potential constants.

The magnetic field influence due to the exchange interaction is described by

$$
H_{\mathrm{ex}}=G_{h} \sigma_{z} \cos \Theta+G_{h} \sigma_{x} \sin \Theta
$$

with

$$
G_{h}=-x_{n} N_{0}\left\langle S_{\mathrm{Mn}}\right\rangle_{H, T} J / 2,
$$

where $x_{n}$ is the concentration of Mn ions, $\left\langle S_{\mathrm{Mn}}\right\rangle$ is the Brillouin function and $J-$ the overlap integral. The Pauli spin operators and the angle between the direction of the magnetic field and the $z$-direction are denoted by $\sigma_{i}$ and $\Theta$, respectively. The $6 \times 6$ matrix representation reads [3]

$$
\begin{aligned}
& H_{\mathrm{ex}}=\left[\begin{array}{cc}
L & R \\
-R & L
\end{array}\right], L=G_{h}\left[\begin{array}{rrr}
1 & 0 & 0 \\
0 & 1 & 0 \\
0 & 0 & -1
\end{array}\right] \cos \Theta, \\
& R=G_{h}\left[\begin{array}{rrr}
1 & 0 & 0 \\
0 & -1 & 0 \\
0 & 0 & -1
\end{array}\right] \sin \Theta .
\end{aligned}
$$

It follows from (6) that only the $x$-component of the magnetic field couples different types of wave functions appearing in (1). 
The $z$-component of the magnetic field gives contributions only in the diagonal components of the Hamiltonian (2). The result is a shift of the energetic position of the states. The wave functions of different states are not mixed.

The splitting of the degenerate states in the $H \| z$ geometry in the presence of the strain is of the same character as in the case without strain. The new feature introduced by the strain is the appearance of a "crossing" of the eigenvalues. The character of the states is not changed. The ordering of the states for high magnetic fields in an arbitrary direction is determined only by the part $H_{\text {ex }}$ of the Hamiltonian (2).

This picture is completely changed by any admixture of the $x$-component of the magnetic field, even in the case of small contributions. Instead of a one-to-one assignment of an energy curve to a definite set of wave functions the character of the wave function is essentially changed due to the coupling. The energy curves do not "cross" any longer, but show a "anti-crossing" behavior.

\section{Envelope-function approximation}

The characteristic features of the interplay of the strain and the magnetic field are preserved if we restrict the calculation to the $\Gamma_{8}$ valence band neglecting the interaction with the spin-orbit split band.

The Hamiltonian for the magnetic field contribution in the Kohn-Luttinger representation reads [4]

$$
H_{\text {mag }}=\left[\begin{array}{cccc}
3 \delta / 2 & \gamma & 0 & 0 \\
\gamma^{*} & \delta / 2 & \alpha & 0 \\
0 & \alpha^{*} & -\delta / 2 & \gamma \\
0 & 0 & \gamma^{*} & -3 \delta / 2
\end{array}\right]
$$

with $\gamma=\mathrm{i} C_{h}^{x} \sqrt{3} / 2, \alpha=\mathrm{i} C_{h}^{x}$ and $\delta=C_{h}^{z}$ and $C_{h}^{x, z}$ being the $x$ - and $z$-magnetic field component dependent prefactors analogous to (6).

Adding to (7) the strain Hamiltonian

$$
H_{\text {str }}=\left[\begin{array}{cccc}
A & 0 & 0 & 0 \\
0 & B & 0 & 0 \\
0 & 0 & B & 0 \\
0 & 0 & 0 & A
\end{array}\right]
$$

with

$$
A=(l+m)\left(\varepsilon_{x x}+\varepsilon_{y y}\right) / 3+m \varepsilon_{z z} \text { and } B=\left[A+2 m\left(\varepsilon_{x x}+\varepsilon_{y y}\right)+l \varepsilon_{z z}\right],
$$

we can calculate the energy values and wave functions. The crossing-anticrossing behavior of the eigenvalue curves and the eigenfunctions is the same as discussed in Sec. 2.

The general features are preserved if we include the interaction between the quantum well and barrier states in the frame of EFA.

Following Ram-Mohan et al. [6, 7] the transfer-matrix method has been used for the calculation of the energy spectrum of a double heterostructure (DHS). The barrier material is doped with $\mathrm{Mn}$, whereas the well is free from manganese ions. 
Nevertheless, there is a pronounced influence of the barrier dopants on the $\Gamma_{8}$ valence bands in the well. The results are shown in Fig. 1. We have assumed that the coupling of the bands is only induced by the magnetic field setting $k_{x}=k_{y}=0$.

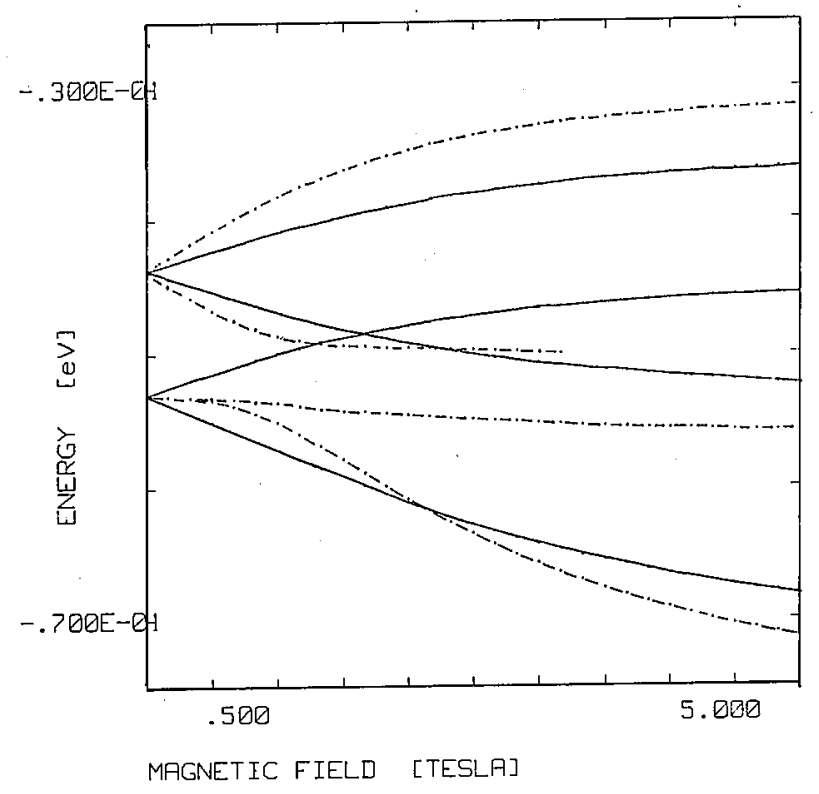

Fig. 1. Energies of the heavy-hole and light-hole states in a strained $Q W$ in dependence on the magnetic field strength and the orientation of magnetic field. The barrier material is unstrained. Width of $\mathrm{QW}-20 \AA$, energetic depth - $0.1 \mathrm{eV}$. Solid line - magnetic field direction parallel to $z$, dashed line - magnetic field direction perpendicular to $z$.

The results are similar to the results obtained on bulk materials. The theoretical curves are in good agreement with the experimental data reported by Goede et al. [1-2].

\section{References}

[1] O. Goede, W. Heimbrodt, Phys. Status Solidi B.146, 11 (1988).

[2] O. Goede, W. Heimbrodt, Th. Koepp, H.-E. Gumlich, B. Lunn, D.E. Ashenford, Phys. Status Solidi B 166, 531 (1991).

[3] S.I. Gubarev, Phys. Status Solidi B 134, 211 (1986).

[4] G.L. Bir, G.E. Picus, Symmetry and Strain-induced Effects in Seminconductors, Wiley, New York 1974.

[5] R.R. Gałązka, J. Kossut, in: Narrow Gap Semiconductors, Physics and Applications, Lecture Notes in Physics, Vol. 133, Ed. W. Zawadzki, Springer-Verlag, Berlin 1980, p. 245.

[6] L.R. Ram-Mohan, K.H. Yoo, R. L. Aggarwal, Phys. Rev. B 38, 6151 (1988).

[7] B. Then, M. Lazzouni, L.R. Ram-Mohan, Phys. Rev. B 45, 1204 (1992). 